\title{
EFFECTS OF DELTA-9-TETRAHYDROCANNABINOL ON THE CARDIOVASCULAR SYSTEM, AND PRESSOR AND BEHAVIORAL RESPONSES TO BRAIN STIMULATION IN RATS
}

\author{
Hiromu KAWASAKI*, Shigenori WATANABE, Ryozo OISHI** \\ and Showa UEKI \\ Department of Pharmacology, Faculty of Pharmaceutical Sciences, \\ Kyushu University, Fukuoka 812, Japan
}

Accepted February 5, 1980

\begin{abstract}
Effects of delta-9-tetrahydrocannabinol ( $\left.4^{9}-\mathrm{THC}\right)$ on the cardiovascular system, and pressor and behavioral responses to brain stimulation were investigated in rats. In urethane-anesthetized rats, intravenous administration of $4^{9}$-THC (1, 2 and $5 \mathrm{mg} / \mathrm{kg}$ ) produced a significant and transient dose-related rise but no fall in blood pressure. $4^{9}$-THC at these doses also produced marked reduction in heart rate which was antagonized by atropine $(0.5 \mathrm{mg} / \mathrm{kg}$ i.v. $)$ or bilateral vagotomy. In freely moving rats with chronically implanted electrodes and arterial cannula implants, intraperitoneal administration of $4^{9}-\mathrm{THC}(4,6$ and $8 \mathrm{mg} / \mathrm{kg})$ induced a rise in blood pressure and marked bradycardia. Abnormal behavior such as catalepsy, walking backwards and pivoting was also observed. $4^{9}$-THC failed to inhibit the pressor responses to electrical stimulation of the posterior hypothalamus and midbrain reticular formation, whereas the drug suppressed the behavioral responses to stimulation of both regions. It is concluded that the cardiovascular effects seen with ${ }^{9}$-THC injection are mainly the result of alteration of the efferent vagal activity by acting on the central nervous system and a more potent influence is exerted on the behavioral changes than on autonomic responses to brain stimulation.
\end{abstract}

Cardiovascular effects of delta-9-tetrahydrocannabinol ( $4^{\vartheta}$-THC), the major psychoactive constituent of marijuana, have been well documented in the rat (1-3), cat (4-6), dog (7-9) and man $(10,11)$. The results obtained in these experiments demonstrate that $4^{9}$ THC induces a hypotension and bradycardia in animals, although heart rate in humans usually increases. Cavero et al. (8) reported that $\Delta^{9}$-THC acted on the central nervous system and produced a decrease in heart rate as a result of the enhancement of vagal activity. They also suggested (7) that the hypotension induced by $4^{9}$-THC was due to a reduction in cardiac output resulting from decreased heart rate.

On the other hand, Vollmer et al. (6) demonstrated that $\Delta^{9}$-THC produced cardiovascular alterations by a central action resulting in a decrease in sympathetic tone. Although there is general agreement that the site of action of $\Delta^{9}$-THC is in the central nervous system, the underlying neural mechanisms involved in cardiovascular alterations

* Present address: Department of Pharmacology, Miyazaki Medical College, Miyazaki, 889-16, Japan.

** Present address: Department of Pharmacology, Faculty of Medicine, Kyushu University, Fukuoka 812, Japan 
by $\Delta^{9}$-THC remain unknown. As for the central action of $\Delta^{9}$-THC, it was reported that $\Delta^{9}$-THC depressed the pressor response induced by electrical stimulation of sympathetic areas such as the hypothalamus and medulla $(4,5)$. However, most of these experiments were performed on anesthetized animals. Recently, Jandhyala and Buckley (12) emphasized that various anesthetic agents exerted considerable influence on the cardiovascular effects of $\Delta^{9}$-THC.

We investigated the effect of $4^{9}$-THC on the cardiovascular system and also on the pressor and behavioral responses to brain stimulation in conscious, freely moving rats and our findings are reported herein.

\section{MATERIALS AND METHODS}

Animals: Male Wistar King A rats weighing between 280-300 g, from Kyushu University Institute of Laboratory Animals, were used. All animals were provided food and water ad libitum and housed in an air conditioned room with a $12 \mathrm{hr}$ light/dark cycle (lights on at $08: 00$ ). After arterial cannula implantation, the animals were transferred to individual cages.

Experiments in anesthetized rats: The animals were anesthetized with urethane $(1.2 \mathrm{~g}$ / $\mathrm{kg}$ i.p.). The femoral artery was cannulated with polyethylene tubing. Blood pressure was measured with a SANEI SOCKI biophysiograph (SYS-180) via a TOYO SOCKI pressure transducer (LPU-0.5-360-0-III). The diastolic pressure plus one-third pulse pressure was regarded to be mean blood pressure. Heart rate was monitored utilizing a NIHON KODEN cardiotachograph (RT-5) triggered by electrocardiogram (ECG) which was amplified with the biophysiograph. Blood pressure and heart rate were simultaneously recorded on a SANEI SOCKI rectigraph (8S). The femoral vein was cannulated with polyethylene tubing for administration of drugs.

Experiments in freely moving rats: For chronic electrode implantation, the rats were anesthetized with pentobarbital $45 \mathrm{mg} / \mathrm{kg}$ i.p. and the head was fixed in a stereotaxic instrument. Bipolar electrodes composed of stainless steel wire of $0.5 \mathrm{~mm}$ in diameter, insulated except for the last $0.5 \mathrm{~mm}$ of the tips were chronically implanted into the posterior hypothalamus (PH) (A: $4.6 \mathrm{~mm}, \mathrm{~L}: 0.5 \mathrm{~mm}, \mathrm{H}:-2.5 \mathrm{~mm}$ ) and the midbrain reticular formation (MRF) (A: $1.5 \mathrm{~mm}, \mathrm{~L}: 1.5 \mathrm{~mm}, \mathrm{H}:-1.5 \mathrm{~mm}$ ) according to the atlas of De Groot (13). Concomitant with this surgery, an ECG lead electrode was chronically implanted and the tip of the electrode was lightly anchored to the muscle of the left thoracic region with fine sutures (14). The remainder was led beneath the skin to emerge at the incision at the head. A reference electrode made of the same stainless steel wire was placed on the skull. Each electrode was soldered to a connector socket and all exposed parts of the electrodes and connector socket itself were fixed on the skull with two screws and dental cement. After the operation 100,000 units of procaine penicillin $\mathrm{G}$ were given i.m. A 10 day recovery period was then allowed.

For chronic cannula implantation, the animal was anesthetized with ether. One end of a cannula (PE10), composed of thin polyethylene tubing (PE10 and PE20), was inserted 
into the abdominal aorta via the left femoral artery. The other end (PE20) was passed beneath the skin to the incision at the dorsum of neck, where it was exteriorized for a distance of $2 \mathrm{~cm}$ and lightly anchored to the surrounding tissue with fine sutures. The arterial cannula was previously filled with heparinized saline ( 500 units $/ \mathrm{ml})$ and plugged with a stainless steel stopper. The complete procedure for the construction and implantation of the arterial cannula was described in our previous report (15). Concomitant with this surgery, a cannula for drug administration, composed of thin polyethylene tubing (PE10 and PE20), was also chronically implanted into the abdominal cavity. The remainder was passed beneath the skin to the neck insicion, exteriorized for a distance of $2 \mathrm{~cm}$, and fixed to the surrounding tissue together with the arterial cannula. After the operation, 100,000 units of procaine penicillin $\mathrm{G}$ were given i.m. To prevent the exposed part of the cannula from being bitten off by other rats, the animals were housed individually. A recovery period of 4 days after the operation was allowed prior to experimentation.

Blood pressure and heart rate in freely moving rats were measured as described previously (14). The arterial cannula stopper was removed and the cannula was attached to a piece of polyethylene tubing connected with a Toyo Socki pressure transducer (LPU-0.5-360-0-III). Arterial blood pressure was measured by a San-ei Socki biophysiograph (SYS-180) via the transducer. The diastolic pressure plus one-third pulse pressure was regarded to be mean blood pressure. Heart rate was measured as beats/min by a Nihon Koden cardiotachograph (RT-5) triggered by ECG which was amplified with a San-ei Socki biophysiograph (SYS-180). Blood pressure and heart rate were simultaneously recorded on a San-ei Socki rectigraph (8S).

The lead wire for electrical stimulation of the brain was connected to the connector socket on the animal's head. Electrical stimulation was applied through a Nihon Koden (MSE-3R) electronic stimulator. After connecting the cannula and lead wire, the animal was transferred to an open-topped plexiglass cage, which was placed in a sound-proof box. Arterial blood pressure and heart rate were measured while observing the behavior of the rat through the window of the sound-proof box. After the animal adapted itself to the cage and was calm, PH and MRF were electrically stimulated. Square wave pulses (1.0 msec, $50 \mathrm{~Hz}$ ) were applied for 5 seconds. The stimulus voltage was increased until steady optimal responses were obtained.

Drugs: Drugs used in the present experiment were as follows: $4^{9}$-THC (extracted from cannabis in the Dept. of Pharmacognosy, Kyushu Univ.) and atropine sulphate (Merck). $\Delta^{9}$-THC was emulsified in $1 \%$ Tween 80 and the emulsion was diluted in $0.9 \%$ saline. In experiments on anesthetized rats, all drugs were diluted with saline and given i.v. In freely moving rats, drugs were administered through the chronically implanted abdominal cannula. The cannula was attached to a polyethylene tubing extension (PE20) which had been previously filled with saline. The other end of the tubing was led to the outside of the soundproof box. The assigned drug was given i.p. through this tubing making it unnecessary to touch the animal. After every administration, the tubing was flushed with saline.

Statistical analysis: Student's $t$-test was used for statistical analysis. 
Histology: After completion of the experiment, the animals were anesthetized with ether and the brains were perfused with $10 \%$ formalin. The brain was removed, fixed, prepared into $50 \mu$ frozen sections, and stained with cresyl violet. Electrode placement was verified histologically.

\section{RESULTS}

Cardiovascular effects of $\Delta^{9}-T H C$ in anesthetized rats

Effects of $\Delta^{9}$-THC on mean blood pressure and heart rate in urethane-anesthetized rats are illustrated in Fig. 1. Intravenous administration of $4^{9}$-THC in doses of 1,2 and $5 \mathrm{mg} / \mathrm{kg}$ induced a significant dose-related transient rise in blood pressure. Peak effect was obtained within $1 \mathrm{~min}$ after the injection. A gradual drop in blood pressure following this transient pressor response was observed with a dose of $5 \mathrm{mg} / \mathrm{kg}$ but not with $1 \mathrm{or} 2 \mathrm{mg} /$ $\mathrm{kg}$. However, there was no significant difference between THC $5 \mathrm{mg} / \mathrm{kg}$ i.v. and control ( $1 \%$ Tween 80 -saline, $1 \mathrm{mg} / \mathrm{kg}$ i.v.). Heart rate was significantly decreased after injection of $4^{9}$-THC in doses of $1-5 \mathrm{mg} / \mathrm{kg}$. The bradycardiac action of $4^{9}$-THC was maximum within 5 min after treatment and lasted for more than $2 \mathrm{hr}$. However, a decrease in heart rate was not observed during the period of maximal pressor response after $\Delta^{9}$-THC injection. Neither blood pressure nor heart rate was altered by i.v. administration of the vehicle.

Intravenous administration of atropine in a dose of $0.5 \mathrm{mg} / \mathrm{kg} 60 \mathrm{~min}$ after $4^{9}$-THC injection reduced the bradycardia induced by all dose levels of $4^{9}$-THC. The hypotension

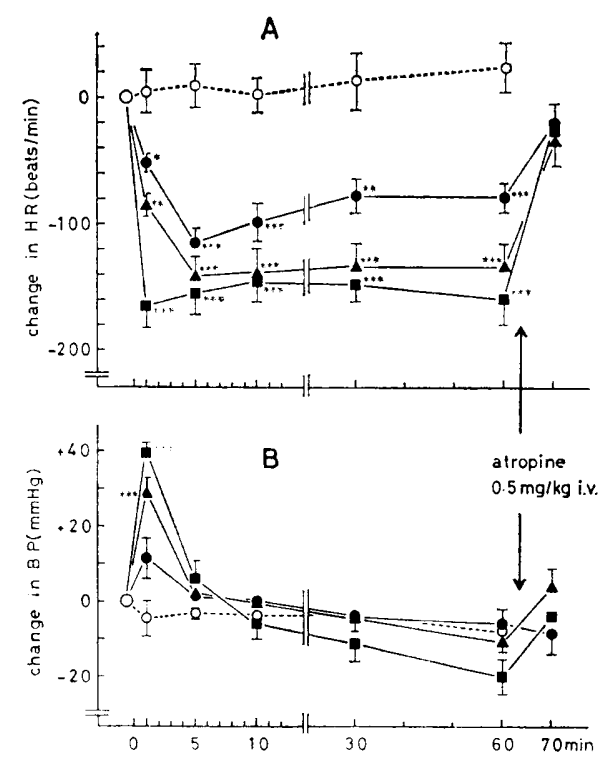

FIG. 1. Effects of $4^{9}$-THC on heart rate (A) and blood pressure (B) in urethaneanesthetized rats. $\bigcirc$ : vehicle $\left(1 \%\right.$ Tween 80 -saline) $1 \mathrm{ml} / \mathrm{kg}$, i.v. $(\mathrm{n}=6)$. $: 4^{9}$-THC $1 \mathrm{mg} / \mathrm{kg}$, i.v. $(\mathrm{n}=8) . \quad \Delta$ : $\Delta^{9}-\mathrm{THC} 2 \mathrm{mg} / \mathrm{kg}$, i.v. $(\mathrm{n}=5) . \quad \boldsymbol{\square}: \Delta^{9}-\mathrm{THC} 5 \mathrm{mg} / \mathrm{kg}$, i.v. $(n=10)$. Vertical bars indicate S.E.M. Atropine sulphate $(0.5 \mathrm{mg} / \mathrm{kg})$ was given i.v. at the arrow $60 \mathrm{~min}$ after $4^{9}$-THC injection. Significant difference from vehicle control: ${ }^{*} \mathrm{p}<0.05,{ }^{* *} \mathrm{p}<0.01,{ }^{* * *} \mathrm{p}<0.001$. 
occurring after the high dose of $\Delta^{9}$-THC was antagonized by atropine. Bilateral vagotomy prior to $\Delta^{9}$-THC $(\mathrm{n}=3)$ or $90 \mathrm{~min}$ after $\Delta^{9}$-THC $(\mathrm{n}=3)$ blocked the bradycardiac effect of $\Delta^{9}-\mathrm{THC}$.

\section{Experiments in freely moving rats}

a) Cardiovascular responses and behavioral changes induced by $\Delta^{9}-T H C$ : When the rat with chronic electrodes and cannula implants was transferred to the plexiglass cage from the home cage, marked exploratory behavior such as rearing, sniffing and searching was observed, and the mean blood pressure and heart rate were high and unstable. Thereafter, as exploratory behavior decreased, mean blood pressure and heart rate gradually fell. Thirty min after being transferred to the plexiglass cage, the rat became calm and continued to sit in one corner of the cage. During this period, mean blood pressure and heart rate were low and very stable. Intraperitoneal administration of $\Delta^{9}$-THC was given through the abdominal cannula without touching the rat and while the animal maintained a sitting

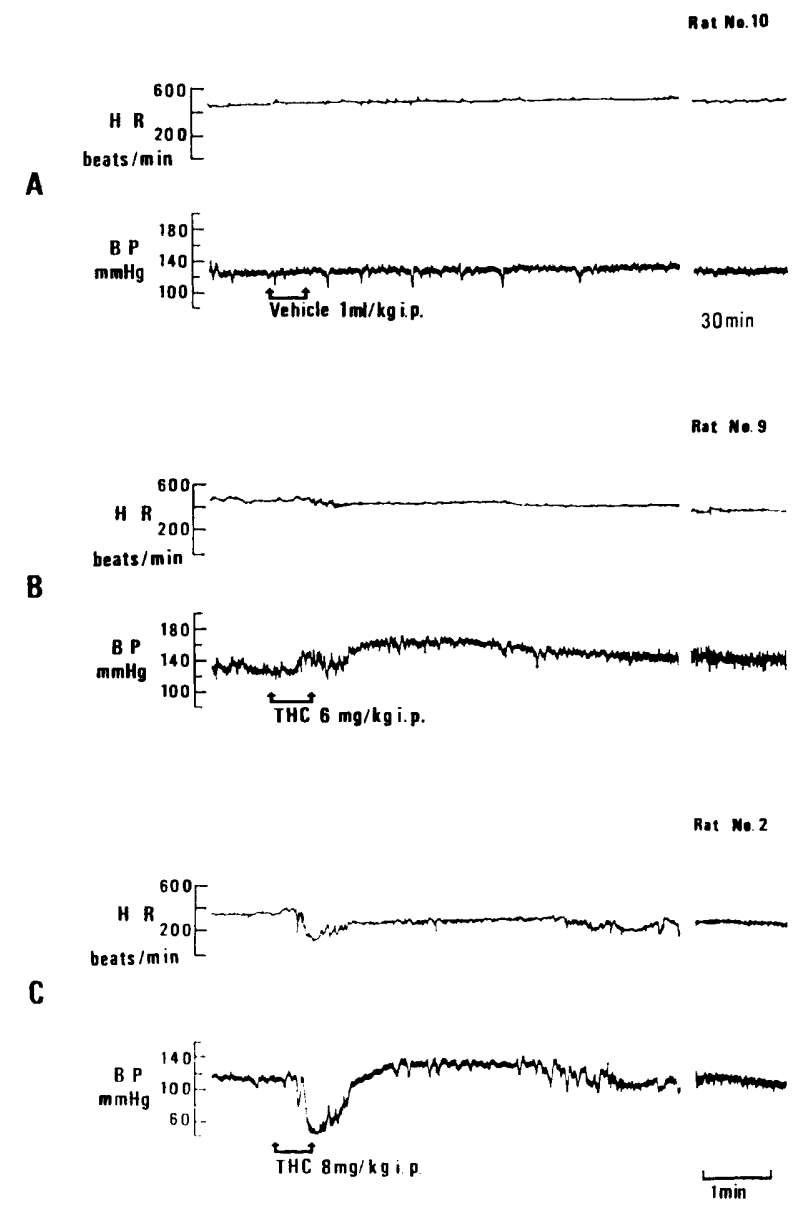

FIG. 2. Cardiovascular response of $d^{9}$-THC in freely moving rats. Vehicle $(1 \%$ Tween 80 -saline) $1 \mathrm{ml} / \mathrm{kg}$ (A), $\Delta^{9}$-THC $6 \mathrm{mg} / \mathrm{kg}$ (B) and $\Delta^{9}$-THC $8 \mathrm{mg} / \mathrm{kg}$ (C) were administered i.p. at the arrow during $30 \mathrm{sec}$. 

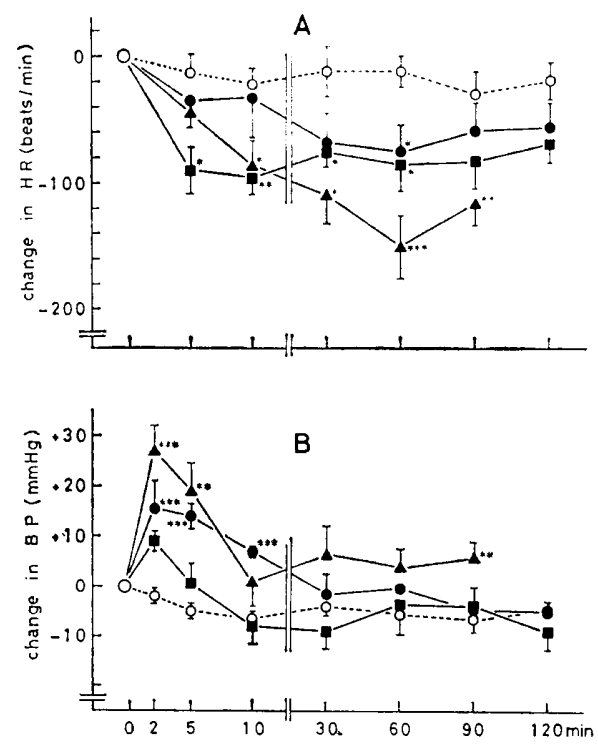

FIG. 3. Effects of $4^{9}$-THC on heart rate (A) and blood pressure (B) in freely moving rats. $\bigcirc$ : vehicle $\left(1 \%\right.$ Tween 80 -saline) $1 \mathrm{ml} / \mathrm{kg}$, i.p. $(\mathrm{n}=6)$, $: \Delta^{9}-\mathrm{THC} 4 \mathrm{mg} / \mathrm{kg}$, i.p. $(\mathrm{n}=4), \boldsymbol{\Delta}: \Delta^{9}$-THC $6 \mathrm{mg} / \mathrm{kg}$, i.p. $(\mathrm{n}=5), \boldsymbol{\square}: \Delta^{9}-\mathrm{THC} 8 \mathrm{mg} / \mathrm{kg}$, i.p. $(\mathrm{n}=6)$. Vertical bars indicate S.E.M. Significant difference from vehicle control: ${ }^{*} \mathrm{p}<0.05$, ${ }^{* *} \mathrm{p}<0.01,{ }^{* * *} \mathrm{p}<0.001$.

posture. Figures 2 and 3 indicate changes in the mean blood pressure and heart rate following the administration of $\Delta^{9}$-THC in freely moving rats. Neither mean blood pressure nor heart rate was altered by the administration of vehicle ( $1 \%$ Tween 80 -saline $1 \mathrm{ml} / \mathrm{kg}$ ) (Fig. 2). $4^{9}$-THC in doses of 4 and $6 \mathrm{mg} / \mathrm{kg}$ but not $8 \mathrm{mg} / \mathrm{kg}$ produced a significant dose-related rise in blood pressure (Fig. 3B). The maximal pressor response was observed within 2-3 min following $\Delta^{9}$-THC and the action lasted for 10-20 min. However, a fall in blood pressure following the pressor response was not seen for $120 \mathrm{~min}$ after $4^{9}$-THC administration. A transient fall of 50-60 mm Hg in blood pressure was observed within $1 \mathrm{~min}$ after administration of $4^{9}$-THC in a dose of $8 \mathrm{mg} / \mathrm{kg}$ (Fig. 2). At this period, the animal frequently exhibited a writhing syndrome.

$\Delta^{9}$-THC in doses of 4,6 and $8 \mathrm{mg} / \mathrm{kg}$ caused a significant reduction in heart rate lasting over $120 \mathrm{~min}$ (Fig. 3A). The bradycardia appeared gradually, and became maximum between 10-60 min after administration of $4^{9}$-THC.

$\Delta^{9}$-THC in doses of 4,6 and $8 \mathrm{mg} / \mathrm{kg}$ reduced spontaneous locomotor activity of the rat and ataxia occurred within $10 \mathrm{~min}$ after administration. After this period, all animals continuously showed catalepsy and such abnormal and unusual behavior as suddenly walking backwards, pivoting and heterophasia. This abnormal behavior occurred most frequently about $30 \mathrm{~min}$ after $4^{9}$-THC administration and lasted for $120 \mathrm{~min}$. Although the blood pressure and heart rate were stable during cataleptic state, during the abnormal behavior, both blood pressure and heart rate was immediately elevated. Even when the animal stopped moving, elevated blood pressure and/or heart rate persisted and gradually returned 


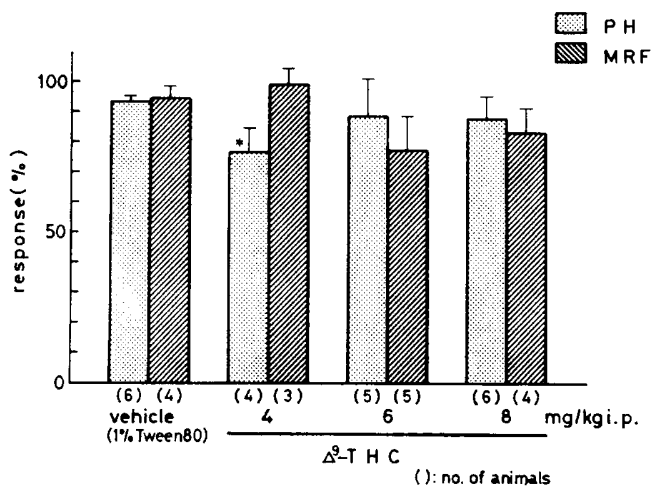

FIG. 4. Changes in the pressor response to electrical stimulation of the posterior hypothalamus $(\mathrm{PH})$ and midbrain reticular formation (MRF) in freely moving rats $30 \mathrm{~min}$ after administration of $\Delta^{9}$-THC. The ordinate represents the percent pressor resoponse. Vertical bars indicate S.E.M. Significant difference from vehicle control: ${ }^{*} \mathrm{p}<0.05$.

to baseline levels after $5 \mathrm{~min}$. During this period, the animals became more aggressive and irritable, and violently vocalized when their back was touched.

b) Effect of $A^{9}-T H C$ on pressor and behavioral responses to PH or MRF stimulation: Electrical stimulation of $\mathrm{PH}$ and MRF elicited an immediate rise in arterial blood pressure in conscious rats. The pressor response to $\mathrm{PH}$ or MRF stimulation consisted of a rapid rise in blood pressure occurring simultaneously with the initiation of stimulation and a return to baseline pressure after the termination of stimulation. Figure 4 shows the changes in pressor response to $\mathrm{PH}$ or MRF stimulation at $30 \mathrm{~min}$ after $4^{9}$-THC administration in freely moving rats. $\quad A^{9}-\mathrm{THC}$ in a dose of $4 \mathrm{mg} / \mathrm{kg}$ produced a small but significant reduction in pressor response to $\mathrm{PH}$ stimulation, while that to MRF stimulation was hardly suppressed by an equivalent dose. $4^{9}$-THC in dose of 6 and $8 \mathrm{mg} / \mathrm{kg}$ slightly reduced the pressor response to both $\mathrm{PH}$ and MRF stimulation but this effect of $4^{9}$-THC was not statistically significant. Generally, the depressive effect of $4^{9}$-THC on the pressor response to brain stimulation was weak and maximal reduction of pressor response by $\Delta^{9}$-THC was about $30 \%$.

In addition to the pressor response, brain stimulation evoked various behavioral changes in conscious rats. PH stimulation elicited sudden arousal, high locomotion with exploration, rearing and circling. MRF stimulation produced flight, escape, circling and running. $\Delta^{9}$ THC in doses of 6 and $8 \mathrm{mg} / \mathrm{kg}$ suppressed behavioral changes to both PH and MRF stimulation. Maximal suppression was observed about $30 \mathrm{~min}$ after $4^{9}$-THC administration and the effect lasted for $90 \mathrm{~min}$.

\section{DISCUSSION}

The present results demonstrated that $\Delta^{9}$-THC produced a dose-related pressor effect in both anesthetized and conscious, freely moving rats despite different routes of administration. In conscious rats, however, $\Delta^{9} \mathrm{THC}$ in a dose of $8 \mathrm{mg} / \mathrm{kg}$ produced only a slight 
pressor response. This may be due to the drastic transient fall in blood pressure seen immediately after administration. The results of the present experiment are supported by the findings of Adams et al. (3), who reported that $\Delta^{8}$ - and $\Delta^{9}$-THC produced a dosedependent rise in blood pressure in anesthetized rats. They suggested that $\Delta^{9}$-THC might have a tyramine-like action on the adrenergic terminals thereby inducing vasoconstrictive action, since pretreatment with phentolamine or reserpine reduced the $\Delta^{9}$-THC-induced pressor response. However, most experiments performed in various species including rats, cats and dogs did not demonstrate a pressor response to $\Delta^{9}$-THC. The discrepancy between our results and those of other investigators may be due to differences in experimental conditions, species and strains of animals employed, the solvent used for $\Delta^{9}$-THC or other factors.

The most significant cardiovascular change induced by $4^{9}$-THC was a decrease in heart rate. Bradycardia occurred in both anesthetized and conscious rats. The present results are in good agreement with the findings obtained by Cavero et al. who reported that the site of action of $\Delta^{9}$-THC was in the central nervous system and the bradycardiac action was due to the increased vagal activity, since the bradycardia induced by $4^{9}$-THC was reduced by atropine and/or bilateral vagotomy in the anesthetized dog (8). We found herein that $4^{9}$-THC-induced bradycardia was antagonized by atropine and/or bilateral vagotomy in the anesthetized rat. Therefore, our results support the suggestion that the reduction in heart rate by $4^{9}$-THC is mainly due to an increase in efferent vagal activity.

On the other hand, a hypotensive action of $\Delta^{9}$-THC was not seen in the conscious rat and only a slight fall in blood pressure occurred with the highest dose of $4^{9}$-THC in the anesthetized rat. It has been reported that $\Delta^{9}$-THC produced a significant and gradual fall in blood pressure in anesthetized rats, cats and dogs. Vollmer et al. (6) proposed that the hypotensive action of $\Delta^{9}$-THC was mainly due to a decrease in sympathetic tone, since the spontaneous discharges in the inferior cardiac nerve were reduced by $\Delta^{9}$-THC in the anesthetized cat. In the anesthetized rat, we found that the slight fall in blood pressure seen after a high dose of $\Delta^{9}$-THC returned to control level concomitant with the antagonism of $\Delta^{9}$-THC-induced bradycardia by atropine. Therefore, as suggested by Cavero et al. (7), the hypotensive action of $\Delta^{9}$-THC may be due in part to a reduction in cardiac output as a result of the decrease in heart rate. There has been no evidence that $\Delta^{9}$-THC has a direct effect on the contractile properties of the myocardium $(7,9)$. On the other hand, in the conscious rat, a decline in blood pressure was not observed after $\Delta^{9}$-THC treatment, although the heart rate was significantly reduced. Thus, the contribution of reduced cardiac output to the hypotension occurring after $\Delta^{9}$-THC administration can be ruled out in light of the results of the present experiment. Recently, Jandhyala and Buckley (12) demonstrated that $4^{9}$-THC produced a marked hypotension in dogs under pentobarbital or urethaneanesthesia, whereas the compound did not produce a significant change in blood pressure in the conscious dog. Thus, the effect of $4^{9}$-THC on the blood pressure is influenced by anesthetic agents.

Adams et al. (3) suggested that the vasoconstrictive action of $\Delta^{9}$-THC, probably 
mediated by enhancement of norepinephrine release from adrenergic terminals, may compensate for the reduction in peripheral vascular resistance, attributable to decreased sympathetic tone. This suggestion is supported by our observation that a hypotensive state was not induced by $\Delta^{9}$-THC.

Hosko and Hardman (4) and Oskoui (5) found that in anesthetized cats $\Delta^{9}$-THC depressed the pressor response induced by the electrical stimulation of central sympathetic areas such as the hypothalamus, medulla and reticular formation. Therefore, they proposed that the cardiovascular effects of $\Delta^{9}$-THC were due to a decrease in sympathetic outflow resulting from the suppression of these brain regions. However, our present results demonstrated that $\Delta^{9}$-THC had only a slight effect on the blood pressure response to $\mathrm{PH}$ and MRF stimulation in the freely moving rat. Based on these findings, it is suggested that the suppression of central sympathetic areas is not essential for the cardiovascular action of $4^{9}$-THC. We speculate that the cardiovascular action of $\Delta^{9}$-THC may be due mainly to the modulation of efferent vagal activity and in part to the decrease in efferent sympathetic outflow.

On the other hand, $\Delta^{9}$-THC considerably depressed the behavioral responses to both PH and MRF stimulation in the conscious rat. Domino et al. (16) demonstrated that the synthetic THC derivative, dimethylheptyl pyran analogue (MHP), elevated the threshold for inducing behavioral responses to stimulation of the midbrain reticular formation, amygdala and posterior hypothalamus in the monkey. Based on these facts, it is conceivable that $\Delta^{9}$-THC acts more strongly on behavioral rather than autonomic responses to brain stimulation. We have reported that psychotropic drugs such as chlorpromazine, diazepam and imipramine little suppressed the behavioral changes to both PH and MRF stimulation in the conscious rat, even when the pressor response was markedly inhibited by these drugs (15). It is interesting that $4^{9}$-THC suppresses the behavioral changes to brain stimulation with less influence on the pressor response, while psychotropic agents have a more potent inhibitory effect on pressor response than that on behavioral changes to brain stimulation.

In conclusion, the present results suggest that the cardiovascular responses to $A^{9}-\mathrm{THC}$ are mainly due to an increase in vagal activity and that $\Delta^{9}$-THC exerts a more potent influence on behavioral responses than on autonomic responses to brain stimulation.

Acknowledgment: We thank Mrs. K. Nagai for excellent technical assistance.

\section{REFERENCES}

1) Milzoff, J.R., Martz, R. and Hanger, R.N.: Evaluation of the depressor response of d-9-tetrahydrocannabinol in the rat. Fedn. Proc. 13, 505 (1972)

2) Graham, J.D.P. AND LI, D.M.F.: Cardiovascular and respiratory effects of cannabis in cat and rat. Brit. J. Pharmacol. 49, 1-10 (1973)

3) Adams, M.D., Earnhardt, J.T., Dewey, W.L. and HaRris, L.S.: Vasoconstrictor actions of $\Delta^{8}$ - and $A^{9}$-tetrahydrocannabinol in the rat. J. Pharmacol. exp. Ther. 196, 649-656 (1976)

4) Hosko, M.J. and Hardman, H.F.: Effect of $4^{9}$-THC on cardiovascular responses to stimulation of vasopressor loci in the neuraxis of anesthetized cats. The Pharmacologist 
13, 296 (1971)

5) Oskoui, M.: Effect of $\Delta^{1}$-tetrahydrocannabinol ( $\Delta^{1}$-THC) on cardiovascular and respiratory system, vasomotor center (VC), sympathetic and vagus nerve discharge activities and myocardial function. Fedn. Proc. 13, 505 (1972)

6) Vollmer, R.R., Cavero, I., Ertel, R.J., Solomon, T.A. and Buckley, J.P.: Role of the central autonomic nervous system in the hypotension and bradycardia induced by (-)- $\Delta^{9}$-trans-tetrahydrocannabinol. J. Pharm. Pharmacol. 26, 186-192 (1974)

7) CAVERo, I. and Jandhyala, B.S.: Hemodynamic effects of $4^{9}$-tetrahydrocannabinol (49-THC). Fedn. Proc. 13, 505 (1972)

8) Cavero, I., Solomon, T., Buckley, J.P. and Jandhyala, B.S.: Studies of the bradycardia induced by (-)- $4^{9}$-trans-tetrahydrocannabinol in anesthetized dogs. Europ. J. Pharmacol. 22, 263-269 (1973)

9) Cavero, I., Lokhandwala, F., Buckley, J.P. and Jandhyala, B.S.: The effect of (-)$\Delta^{9}$-trans-tetrahydrocannabinol on myocardial contractility and venous return in anesthetized dogs. Europ. J. Pharmacol. 29, 74-82 (1974)

10) Weiss, J.L., Watanabe, A.M., Lemberger, L., Tamarkin, N.R. and Cardon, P.V.: Cardiovascular effects of delta-9-tetrahydrocannabinol in man. Clin. Pharmacol. Ther. 13, 671-684 (1972)

11) Bachman, J.A., Benowitz, N.L., Herning, R.I. and Jones, R.T.: Dissociation of autonomic and cognitive effects of THC in man. Psychopharmacology 61, 171-175 (1979)

12) Jandhyal.A, B.S. AND Buckley, J.P.: Influence of anesthetic agents on the effects of $\Delta^{9}$ tetrahydrocannabinol on the heart rate and blood pressure of the mongrel dog. Europ. J. Pharmacol. 44, 9-16 (1977)

13) DE Groor, J.: The rat forebrain in stereotaxic coordinates. Verh. K. ned. Alad. Wet. 62, 3-41 (1959)

14) Kawasaki, H., Watanabe, S. and Ueki, S.: Changes in blood pressure and heart rate following bilateral olfactory bulbectomy in rats. Physiol. Behav. 24, 51-56 (1980)

15) Kawasaki, H., Watanabe, S. AND Ueki, S.: Effects of psychotropic drugs on pressor and behavioral responses to the brain stimulation in unrestrained, unanesthetized rats. Biochem. Pharmacol. Behav. 10, 907-915 (1979)

16) Domino, E.F., Hardman, H.F. and Seevers, M.H.: Central nervous system actions of some synthetic tetrahydrocannabinol derivatives. Pharmacol. Rev. 23, 317-336 (1971) 